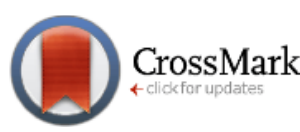

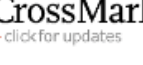

\section{Hepatoprotective effect of a polyherbal formulation and ascorbic acid in paracetamol induced hepatic damage in rabbits}

\section{Muhammad Fiaz ${ }^{1, *}$, Naghma Fiaz ${ }^{2}$, Lubna Shakir ${ }^{1}$, Alamgeer ${ }^{3}$, Waseem Mehmood4, Ghulam Mustafa5, Abdul Rauf', Komal Najam ${ }^{1}$}

\author{
${ }^{1}$ Faculty of Pharmacy, Hajvery University, Lahore, Pakistan \\ 2University of the Punjab, Lahore, Pakistan \\ ${ }^{3}$ Faculty of Pharmacy, University of Sargodha, Sargodha, Pakistan \\ ${ }^{4}$ College of Pharmacy, University of the Punjab, Lahore, Pakistan \\ ${ }^{5}$ Department of Pathology, University of Veterinary and Animal Sciences, Lahore, \\ Pakistan \\ ${ }^{6}$ Department of Pathology, Nawaz Sharif Medical College, University of Gujrat, Gujrat, \\ Pakistan
}

\section{*For correspondence:}

Fiaz.di139@yahoo.com

Competing interests: The authors declare that no competing interests exist.

Received: 11 March 2017

Accepted: 13 April 2017

Published: 27 April 2017

Copyright The Author(s) 2017. This article is published with open access by BioMedPress (BMP).

This article is distributed under the terms of the Creative Commons Attribution License (CC-BY 4.0) which permits any use, distribution, and reproduction in any medium, provided the original author(s) and the source are credited.

\section{Abstract}

Background: The hepatoprotective effect of a polyherbal formulation was evaluated in female rabbits. The herbal formulation was used alone and in combination with ascorbic acid (AsAc) in animals with paracetamol (ParCM)-induced hepatic damage. Methods: The study design included five groups, each comprised of five animals. Group A was the control group (untreated; given only routine diet) while group B was given a single oral dose of ParCM $(2 \mathrm{~g} / \mathrm{kg})$ on day 9. Groups C, D and E were pretreated with polyherbal formulation (PoHF; 500 mg/kg), ascorbic acid (AsAc; $200 \mathrm{mg} / \mathrm{kg}$ ) and PoHF (500 mg/kg) combined with ascorbic acid (AsAc; $200 \mathrm{mg} / \mathrm{kg}$ ), respectively for 9 consecutive days. On the last day (day 9), after 30 minutes of routine treatments, a single dose of ParCM (2 g/ $\mathrm{kg}$ ) was administered in groups C, D and E. Animals were sacrificed 24 hours after the last treatment. Blood and liver samples were collected from all animals. Serum was separated from the blood samples and subjected to biochemical tests for liver biomarker analysis. The biomarkers included alanine aminotransferase (ALT), aspartate aminotransferase (AST), alkaline phosphatase (ALP) and total bilirubin. Elevation of enzyme markers was considered an indicator of hepatocellular injury. Results: Serum levels of liver enzymes and total bilirubin were elevated significantly in group B when compared to group A. The level of serobiochemicals significantly dropped in group C but increased significantly in group $D$, as compared to group $B$. 
No statistically significant effect on liver enzymes was observed in group $E$ when compared to group B. Groups D and E showed a significantly higher level of serological parameters as compared to group C. The biochemical findings were further corroborated with histopathological analyses of the liver tissue samples. Histopathological examination of the livers of rabbits in group A showed normal hepatic cell architecture. However, groups B and D revealed severe congestion of the central vein and sinusoids, periportal fibrosis and infiltration of inflammatory cells; these parameters were of mild and mostly moderate severity in groups $C$ and $E$, respectively. The histopathological findings strongly supported the results of the biochemical analyses. Conclusion: Thus, our study herein demonstrates that herbal formulation remains an effective means to ameliorate ParCM-induced elevation of serum biochemical parameters and changes to the liver histology. Ascorbic acid induced deteriorating effects in a ParCM-intoxicated rabbit animal mode. Moreover, combination of herbal product and ascorbic acid failed to yield liver protecting effects in ParCMpoisoned animals. The damaging effects of ascorbic acid might be attributed to its prooxidant attitude, as reported by many research studies. To expand on these findings further studies are warranted, including evaluating other hepatotoxicity inducers (besides ParCM), testing different doses of ascorbic acid and ParCM, and testing a greater number of animals along with other animal models.

\section{Keywords}

Ascorbic acid, Hepatoprotective, Paracetamol, Polyherbal formulation

\section{Introduction}

The liver is a dynamic organ important for a multitude of functions, namely the assimilation and conversion of nutrient materials absorbed from the gastrointestinal tract to a more useful form of energy, and the neutralization and removal of a number of drugs, xenobiotics and various other substances from the body (Nadeem et al., 1997). The liver is a vibrant organ in the human body, playing an indispensable job of detoxification of countless endogenous and exogenous harmful substances (Yang et al., 2010). As the liver is consistently exposed to environmental poisonous elements (such as drugs), various hepatic disorders can result; liver damage can lead to hepatic failure and eventual death (Mukherjee et al., 2006).

Modern drugs are not suitable for liver diseases due to the high rate of undesirable effects. It is, therefore, necessary to search for alternative remedies (Kang, 2002). There are some natural plants which show excellence at preventing, treating and curing liver diseases, while imposing only a few side 
effects. The hepatoprotective class of therapeutic regimens possesses the competence to protect liver damage and revitalize hepatocytes. International agencies, such as the World Health Organization, have insisted on the evaluation of herbs and herbal products by advanced scientific standards (Neraliya and Gaur, 2004). About 600 herbal drugs are being marketed all over the world for hepatoprotective effects (Sharma et al., 1991). However, only a limited number of formulations have been scientifically qualified for their prescribed use. Examples of the compound formulations which have been scientifically validated for their intended use in liver diseases are: Amalkadi Ghrit, an herbal formulation (Achliya et al., 2004), Liv-52 Syrup \& Livomyn Suspension (Sapakal et al., 2008), Liv-52, Livergen, Livokin, Octogen, Stimuliv and Tefroliv liquid formulations (Girish et al., 2009), Livergen (a polyherbal liquid formulation) (Arsul et al., 2011), Khamira Gaozaban Ambri Jadwar Ood Saleeb Wala (Akhtar et al., 2013), Herbal drug (Feroz and Khan, 2013), Herbal preparations F-I, F-II \& F-III (Sivakumar et al., 2014), and Virgoliv Syrup (Ingawale et al., 2015).

Paracetamol (ParCM) is an excellent analgesic and antipyretic. The toxicity of ParCM is related to its biotransformation which mostly follows three steps: sulfation, glucuronidation and oxidation (Mitchell et al., 1974). Oxidation is the most crucial step as far as hepatic toxicity is concerned (Jollow et al., 1973). A small quantity of ParCM undergoes oxidation by cytochrome P450 2E1 to produce an extremely reactive component, $\mathrm{N}$-acetyl-P benzoquinoneimine (NAPQI) (Vermeulen et al., 1992). When ParCM is used in therapeutic doses, NAPQI is conjugated by hepatic glutathione (GSH) to produce a water-soluble product called mercapturic acid (Lee et al., 1996). The rate of production of GSH counterbalances the toxicity of NAPQI synthesized as a consequence of ParCM oxidation so there is no damage to the hepatocytes so long as this balance is maintained (Sabina et al., 2009). As higher doses of ParCM are administered, generation of GSH in the liver cells is diminished after a few hours and hepatic intoxication is evident when the level of GSH in liver is less than $30 \%$ of the normal value (Makin and Williams, 1997). Uninhibited NAPQI becomes noxious by binding to macromolecules, including cellular proteins (Vermeulen et al., 1992). Ascorbic acid (AsAc) is a popular antioxidant that can act as pro-oxidant under certain conditions (Duarte and Lunec, 2005).

Polyherbal formulation (PoHF) has been claimed to be effective in liver inflammation and other liver aliments. The composition of PoHF consists of: Zingiber officinale (Ginger), Peganum harmala (Wild Rue), Cassia angustifolia (Senna), and Operculina turpethum (Turpeth). PoHF contains flavonoids \& phenolic compounds (present in Zingiber officinale) (Ghasemzadeh et al., 2010), alkaloids (present in Peganum harmala) (Monsef et al., 2004), and glycosidic resins and beta-sitosterol (present in Operculina turpethum) (Iweala et al., 2011). The aim of this study was to validate the hepatoprotective effects of PoHF, which is currently being used without any scientific authentication. In our study, PoHF was used alone and in combination with ascorbic acid (AsAc) in ParCM-induced hepatic damage in rabbits. PoHF was evaluated on the basis of various biochemical and histopathological parameters. 


\section{Materials - Methods}

\section{Polyherbal formulation (PoHF)}

The PoHF evaluated in our study is comprised of parts from four medicinal plants, namely Zingiber officinale (Ginger), Peganum harmala (Wild Rue), Cassia angustifolia (Senna) and Operculina turpethum (Turpeth). The product is in semisolid dosage form and each 10 grams of the herbal formulation contains Zingiber officinale (2 g), Peganum harmala (2 g), Cassia angustifolia (2 g) and Operculina turpethum (2 g). PoHF was obtained from the local manufacturer of the herbal product.

\section{Animals}

Twenty-five female rabbits of local breed (Oryctolagus cuniculus) of particular age (2.5-3.5 months) and weight (550-750 g) were used in the study. They were fed a balanced diet. Animals were kept at light and dark cycle (12/12 h) at room temperature $\left(25 \pm 2^{\circ} \mathrm{C}\right)$ and relative humidity $(60-65 \%)$. Animals were acclimatized to the atmosphere of their environment for one week prior to the start of treatment. Protocols for animal use were approved by the Committee on Animal Ethics, Hajvery University, Lahore, Pakistan.

\section{Experimental Design}

Animals were randomly divided into 5 groups; each group was comprised of 5 animals. The duration of the study was 9 days and the administration of drugs was through oral route.

Group A: This group served as negative control (was given only routine diet).

Group B: Animals in this group were administered with a single dose of ParCM $(2 \mathrm{~g} / \mathrm{kg})$ on the $9^{\text {th }}$ day of treatment using $1 \% \mathrm{CMC}$ as a vehicle.

Group C: Animals in this group were administered with a single dose of PoHF (500 mg/kg; dispersed in normal saline) daily for 9 days, followed by a single dose of ParCM $(2 \mathrm{~g} / \mathrm{kg})$ on the $9^{\text {th }}$ day, after 30 minutes of routine treatment.

Group D: Animals in this group were administered with a single dose of AsAc (200 mg/kg; dissolved in distilled water) for 9 successive days followed by a single dose of $\operatorname{ParCM}(2 \mathrm{~g} / \mathrm{kg})$ on the $9^{\text {th }}$ day, after 30 minutes of routine treatment.

Group E: Animals in this group were administered with PoHF $(500 \mathrm{mg} / \mathrm{kg}$; dispersed in normal saline) \& AsAc (200 mg/kg; dissolved in distilled water) for 9 days, and then a single dose of $\operatorname{ParCM}(2 \mathrm{~g} / \mathrm{kg})$ on the last day, after 30 minutes of routine treatment. 


\section{Determination of Liver Enzymes (ALT, AST and ALP) \& Total Bilirubin}

Animals were sacrificed at 24 hours after the last treatment. Samples of blood were collected in blood collection glass tubes from the control and treated rabbits. Blood samples were stored at room temperature for 30 minutes for coagulation and then centrifuged at $4000 \mathrm{rpm}$ for 8 minutes to separate the serum, which was collected in aliquots for biochemical testing. Alanine aminotransferase (ALT), aspartate aminotransferase (AST) and alkaline phosphatase (ALP) levels were analyzed by Micro Lab 300 (Semi-automated clinical chemistry analyzer) using standard kits (Merck, France). Total Bilirubin was measured using standard kits (DiaSys Diagnostic Systems, Germany).

\section{Tissue Processing of Liver Samples for Histopathological Evaluations}

The liver of each rabbit was excised and extra connective tissues adhered to the organ were removed. Organs were washed with distilled water and small pieces of liver were cut and immersed immediately in 10\% Neutral Buffered Formalin (NBF).

Standard techniques for Fixation, Washing, Dehydration, Clearing, Embedding, Sectioning and Staining (Carleton et al., 1967) of liver tissue samples were adopted. The Hematoxylin \& Eosin stained slides were then examined under different resolutions of microscope in a photographic facility and photomicrographs were taken.

\section{Statistical Analysis}

One-way ANOVA followed by post hoc, Tukey HSD were used to determine the statistical differences between the means of study groups, using the SPSS computer software (version 23). The level of significance was set at $p<0.05$.

\section{Results}

\section{Analysis of Biochemical Parameters}

For group $B$, the administration of a single dose of ParCM increased the serum concentrations of ALT, AST, ALP and total bilirubin to significantly $(P<0.05)$ higher values as compared to group $A$ (the negative control group). Pretreatment with PoHF in group $C$ caused liver enzymes and total bilirubin to drop to significantly $(P<0.05)$ lower levels as compared to group B. Oral administration of AsAc in group $D$ caused an elevation of serobiochemicals to a significantly $(P<0.05)$ higher number in comparison to group $B$. The level of serobiochemicals in group $\mathrm{D}$ was also significantly higher than group $\mathrm{C}$.

PoHF and AsAc were co-administered in group E. Comparison of hepatic enzymes in groups $B \& E$ showed a statistically non-significant $(P>0.05)$ relation. 
Serum concentrations of liver enzymes in group $E$ rose to a significantly higher level $(P<0.05)$ when compared to group $C$ (Table 1; Figs. 1, 2, 3, 4).

Table 1. Effect of treatments on serum ALT, AST, ALP and Total bilirubin in Par-CM induced liver damage

\begin{tabular}{|c|c|c|c|c|c|}
\hline Groups & Treatments & ALT (IU/L) & AST (IU/L) & ALP (IU/L) & $\begin{array}{c}\text { Total } \\
\text { bilirubin } \\
\text { (mg/dL) }\end{array}$ \\
\hline A & Negative control & $37.20 \pm 3.19$ & $26.40 \pm 3.57$ & $71.60 \pm 2.19$ & $0.42 \pm 0.04$ \\
\hline B & Par-CM $(2 \mathrm{~g} / \mathrm{kg})$ & $68.20 \pm 3.27^{\#}$ & $68.20 \pm 5.54^{\#}$ & $159.60 \pm 3.65^{\#}$ & $1.52 \pm 0.04^{\#}$ \\
\hline C & $\begin{array}{l}\text { Po-HF }(500 \mathrm{mg} / \mathrm{kg}+ \\
\text { Par-CM }(2 \mathrm{~g} / \mathrm{kg})\end{array}$ & $46.40 \pm 2.40^{\star}$ & $39.00 \pm 1.58^{*}$ & $83.20 \pm 3.03^{*}$ & $0.54 \pm 0.05^{*}$ \\
\hline D & $\begin{array}{l}\text { As-Ac }(200 \text { mg/kg) } \\
+ \text { Par-CM }(2 \mathrm{~g} / \mathrm{kg})\end{array}$ & $78.40 \pm 4.39^{\star}, \wedge$ & $82.40 \pm 3.05^{*} \wedge$ & $191.00 \pm 5.78^{* \wedge}$ & $1.76 \pm 0.05^{*}$ \\
\hline E & $\begin{array}{c}\text { Po-HF }(500 \mathrm{mg} / \mathrm{kg})+ \\
\text { As-Ac }(200 \mathrm{mg} / \mathrm{kg}) \\
+ \text { Par-CM }(2 \mathrm{~g} / \mathrm{kg})\end{array}$ & $60.60 \pm 5.98^{\wedge}$ & $59.00 \pm 8.27^{\wedge}$ & $145.20 \pm 16.11^{\wedge}$ & $1.44 \pm 0.05^{\wedge}$ \\
\hline
\end{tabular}

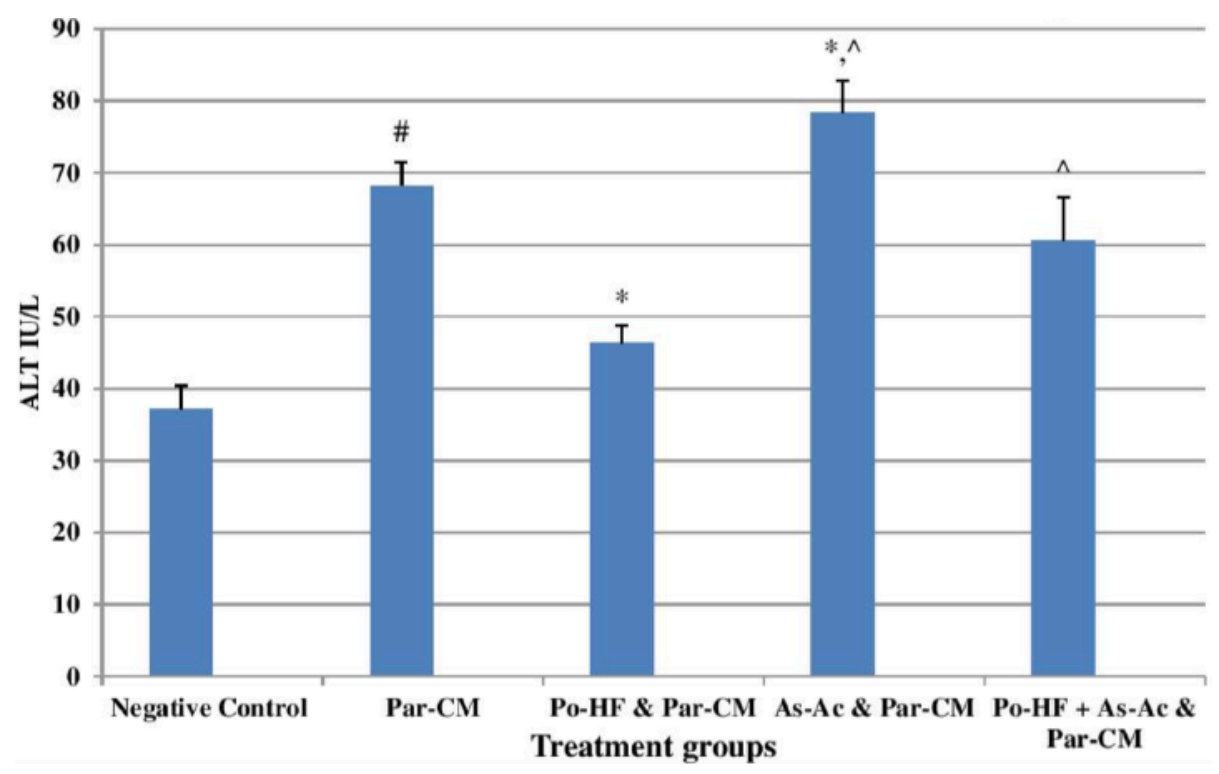

Figure 1. Effect of treatments on serum ALT in Par-CM induced liver damage. Each bar represents the Mean \pm SD of five rabbits. The sign (\#) indicates a significant difference at $p<0.05$ level from the negative control group. $\left(^{\star}\right)$ indicates a significant difference at $\mathrm{p}<0.05$ from the Par-CM intoxicated group. $(\wedge)$ indicates a significant difference at $p<0.05$ from only Polyherbal formulation (Po-HF) treated group. Po-HF \& Ascorbic acid (As-Ac) were given for 9 days and Par-CM for the last day only. 


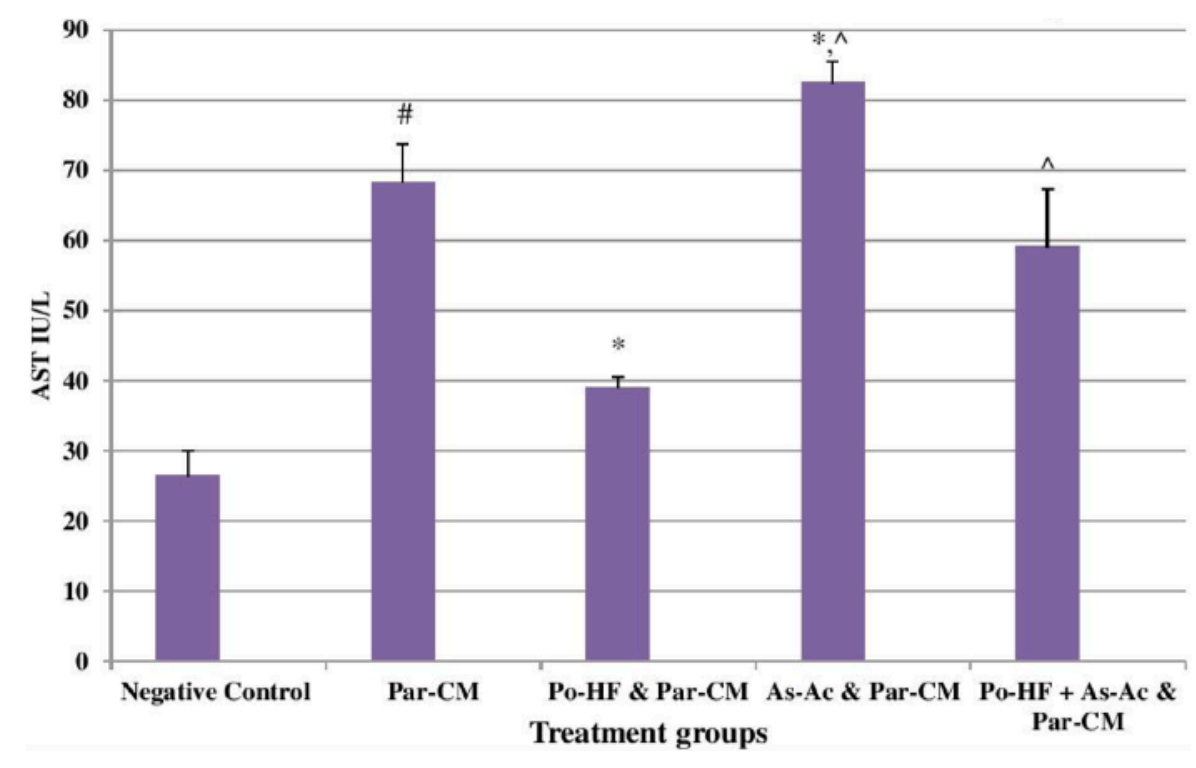

Figure 2. Effects of treatments on serum AST in Par-CM induced liver damage. Each bar represents the Mean \pm SD of five rabbits. The sign (\#) indicates a significant difference at $p<0.05$ level from the negative control group. $\left(^{\star}\right)$ indicates a significant difference at $p<0.05$ from the Par-CM intoxicated group. $(\wedge)$ indicates a significant difference at $p<0.05$ from only Polyherbal formulation (Po-HF) treated group. Po-HF \& Ascorbic acid (As-Ac) were given for 9 days and Par-CM for the last day only.

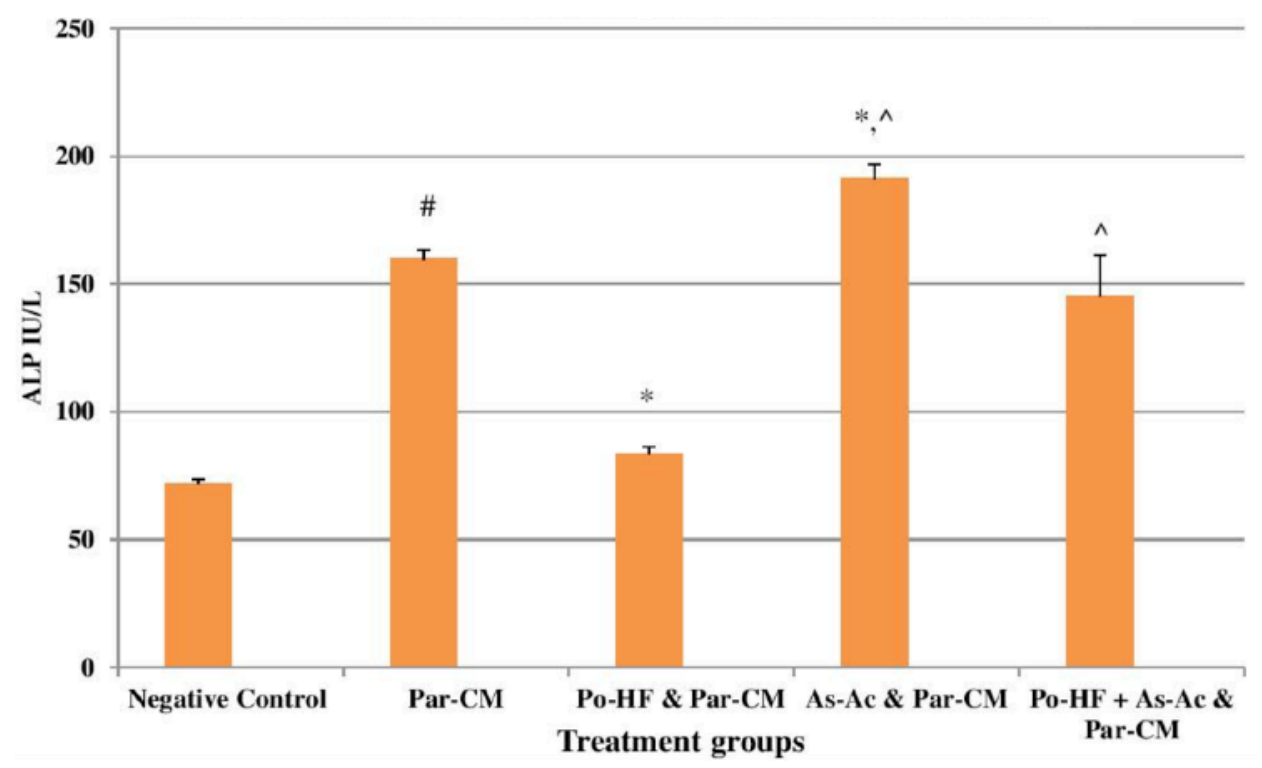

Figure 3. Effects of treatments on serum ALP in Par-CM induced liver damage. Each bar represents the Mean \pm SD of five rabbits. The sign (\#) indicates a significant difference at $p<0.05$ level from the negative control group. $\left(^{\star}\right)$ indicates a significant difference at $p<0.05$ from the Par-CM intoxicated group. $(\wedge)$ indicates a significant difference at $p<0.05$ from only Polyherbal formulation (Po-HF) treated group. Po-HF \& Ascorbic acid (As-Ac) were given for 9 days and Par-CM for the last day only. 


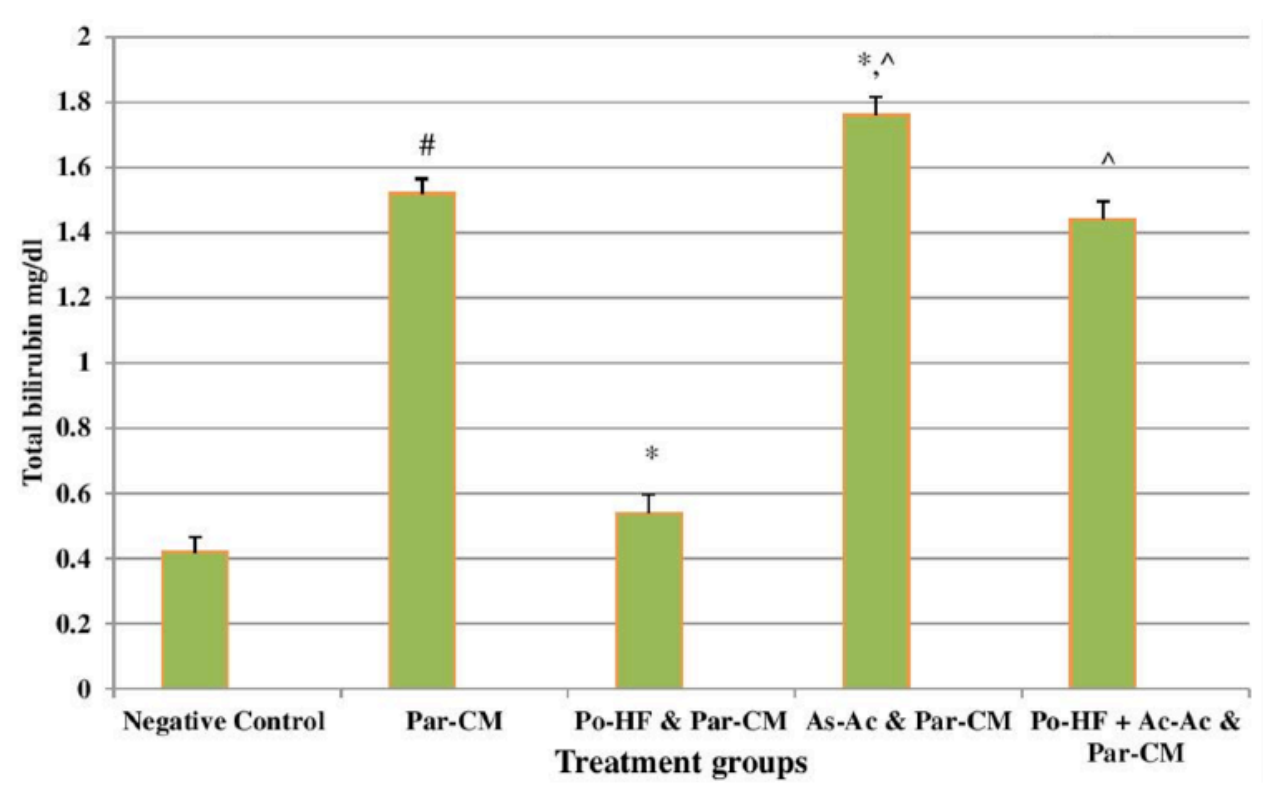

Figure 4. Effects of treatments on T. Bilirubin in Par-CM induced liver damage. Each bar represents the Mean \pm SD of five rabbits. The sign (\#) indicates a significant difference at $p<0.05$ level from the negative control group. $\left(^{\star}\right)$ indicates a significant difference at $\mathrm{p}<0.05$ from the Par- $\mathrm{CM}$ intoxicated group. $(\wedge)$ indicates a significant difference at $p<0.05$ from only Polyherbal formulation (Po-HF) treated group. Po-HF \& Ascorbic acid (As-Ac) were given for 9 days and Par-CM for the last day only.

\section{Histopathological Findings}

Hematoxylin \& Eosin stained sections of liver tissues of rabbits from all study groups, except negative control group A, exhibited various histopathological changes as seen by microscopy. Histopathological analysis revealed that group A (negative control) had the most prominent central vein. There was a severe congestion of the central vein in groups $B \& D$, moderate congestion in group $E$, and mild congestion in group $C$ (Fig. 5). Microscopic examination of paraffin embedded and H\&E stained slides of rabbit livers showed that there was no sinusoidal congestion in group $A$ while there was a severe, mild, severe and moderate congestion of sinusoids observed in groups $B, C, D$ and $E$, respectively (Fig. 6). Microscopic evaluation of processed slides of rabbit liver tissues showed no inflammatory cell infiltration for group A. However, there was a severe degree of infiltration of inflammatory cells for groups $B$ \& $D$, a moderate level of infiltration for group $E$, and a mild level of infiltration for group C (Fig. 7). Microscopy of liver sections was represented by different levels of periportal fibrosis; Group A had a normal periportal area, groups B, D and E showed a severe collection of connective tissues around the portal area, while group C showed no presence of fibrosis (Fig. 8). 


\section{Biomedical}

Research \& Therapy
ISSN: 2198-4093 www.bmrat.org
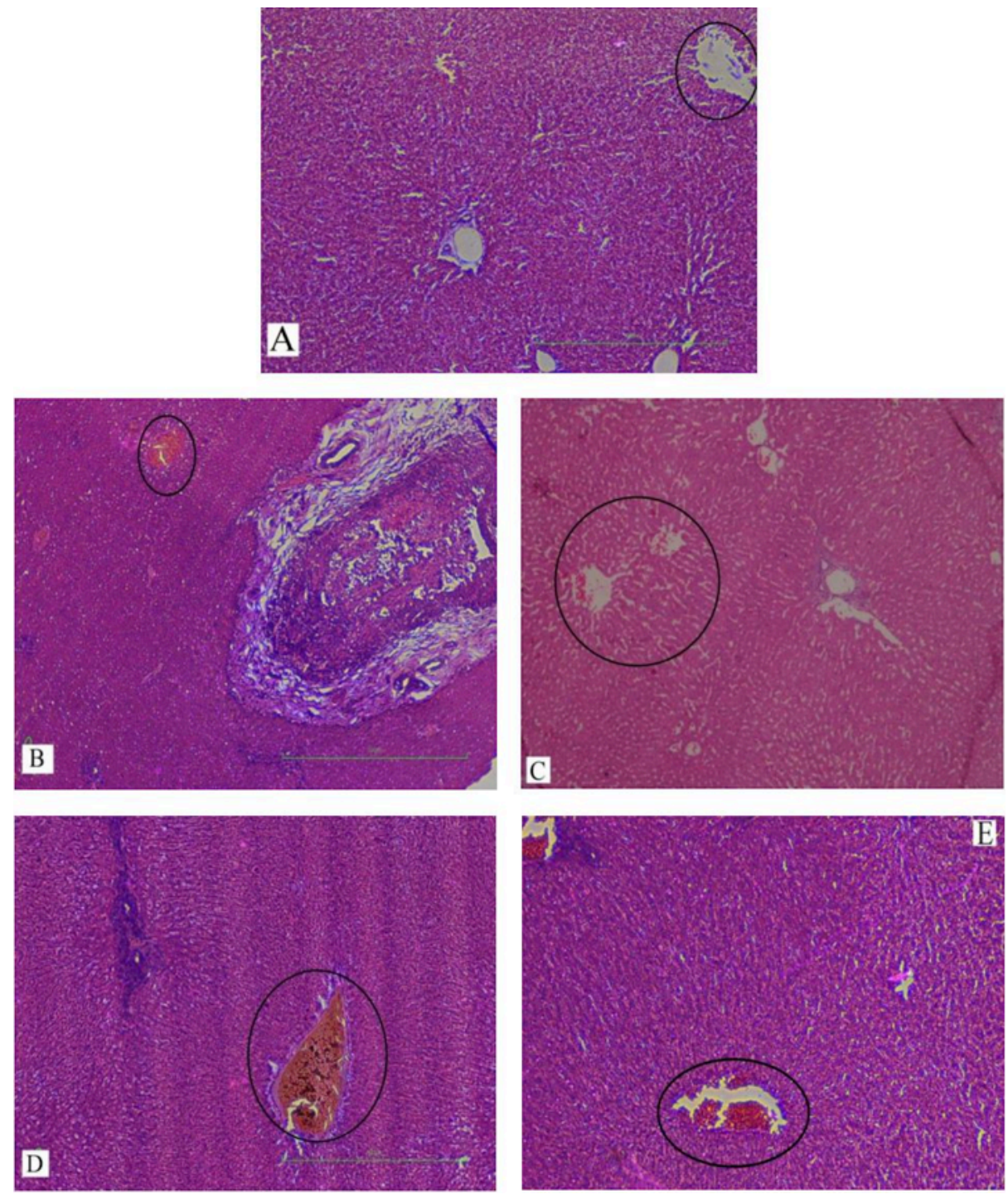

Figure 5. H\&E staining of liver sections. H\&E stained sections of liver tissue shows severe (group B, Par-CM intoxicated), mild (group C, Po-HF \& Par-CM), severe (group D, As-Ac \& Par-CM), moderate (group E, Po-HF + As-AC \& Par$\mathrm{CM}$ ) congestion of central vein. Group (A, negative control) shows normal central vein. The degree of congestion of central vein represents the extent of hepatic damage.

\section{Discussion}

ParCM-induced hepatotoxicity is an authentic method for evaluation of hepatoprotective effects of various medicinal plants and herbal formulations. Numerous studies have demonstrated that at high doses, ParCM produces hepatic harm or necrosis (Vermeulen et al., 1992). Bonkovsky et al. (1994) reported that ParCM, in a single overdose or continual low doses, causes 
hepatic damage (Bonkovsky et al., 1994). The extent of liver damage can be accessed from the level of enzymes like ALT, AST and ALP in the serum, as indicators of the physiological status of the liver. Liver enzymes residing in hepatic cells are released in the bloodstream after cellular insult by hepatotoxic dosage of ParCM (Parmar et al., 2010). When hepatocytes are injured, the permeability of the cell membrane and transport mechanisms are altered, resulting in seepage of enzymes from the cells leading to an increase in serum levels of these biomarkers (Jain and Singhai, 2012; Raja et al., 2007).
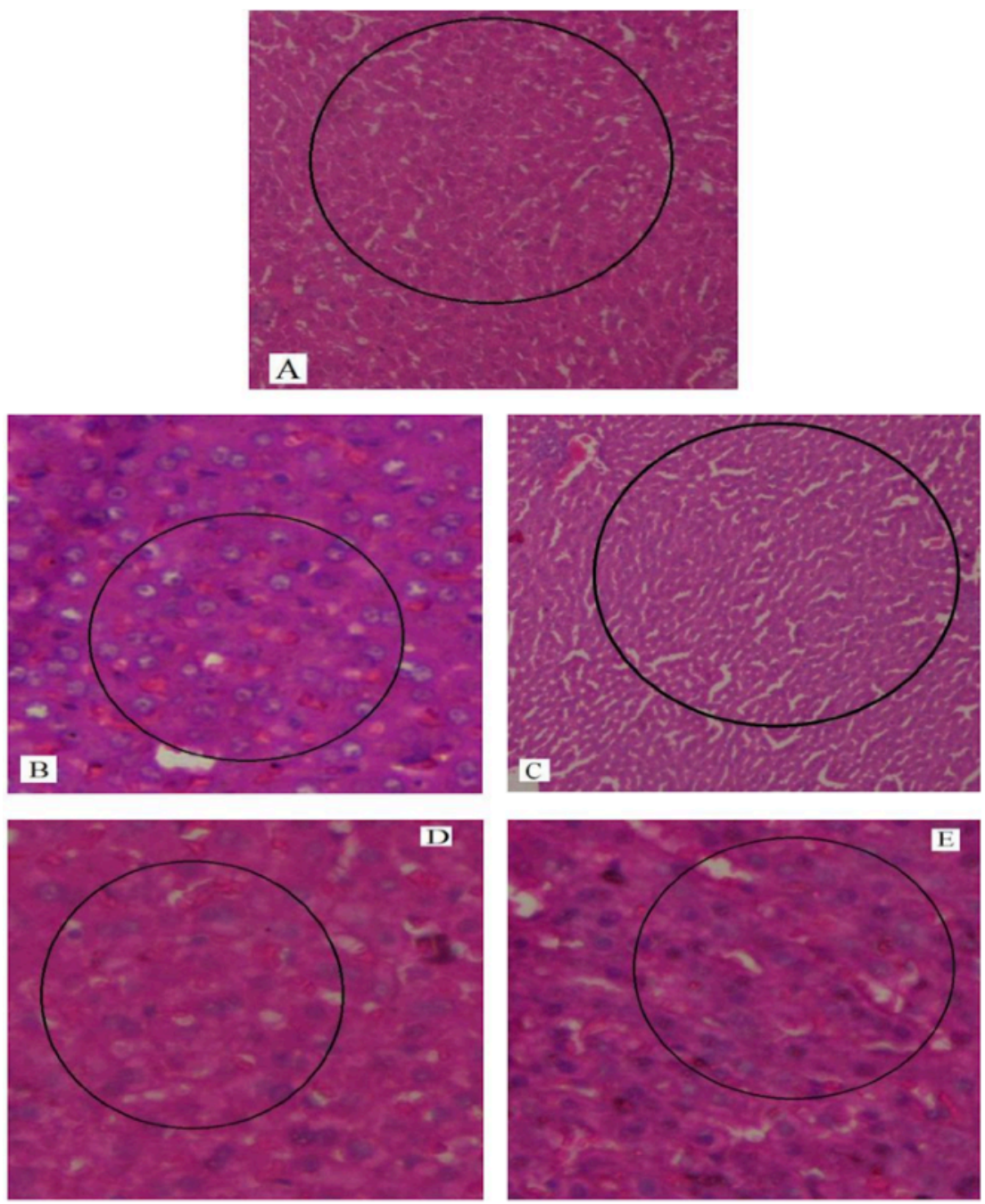

Figure 6. H\&E staining of liver sections. H\&E stained sections of liver tissue shows severe (group B, Par-CM intoxicated), mild (group C, Po-HF \& Par-CM), severe (group D, As-Ac \& Par-CM), moderate (group E, Po-HF + As-AC \& Par$\mathrm{CM}$ ) congestion of sinusoids. Group (A, negative control) shows normal sinusoids. The intensity of sinusoidal congestion illustrates the level of liver damage. 


\section{Biomedical}

Research \& Therapy
ISSN: 2198-4093 www.bmrat.org
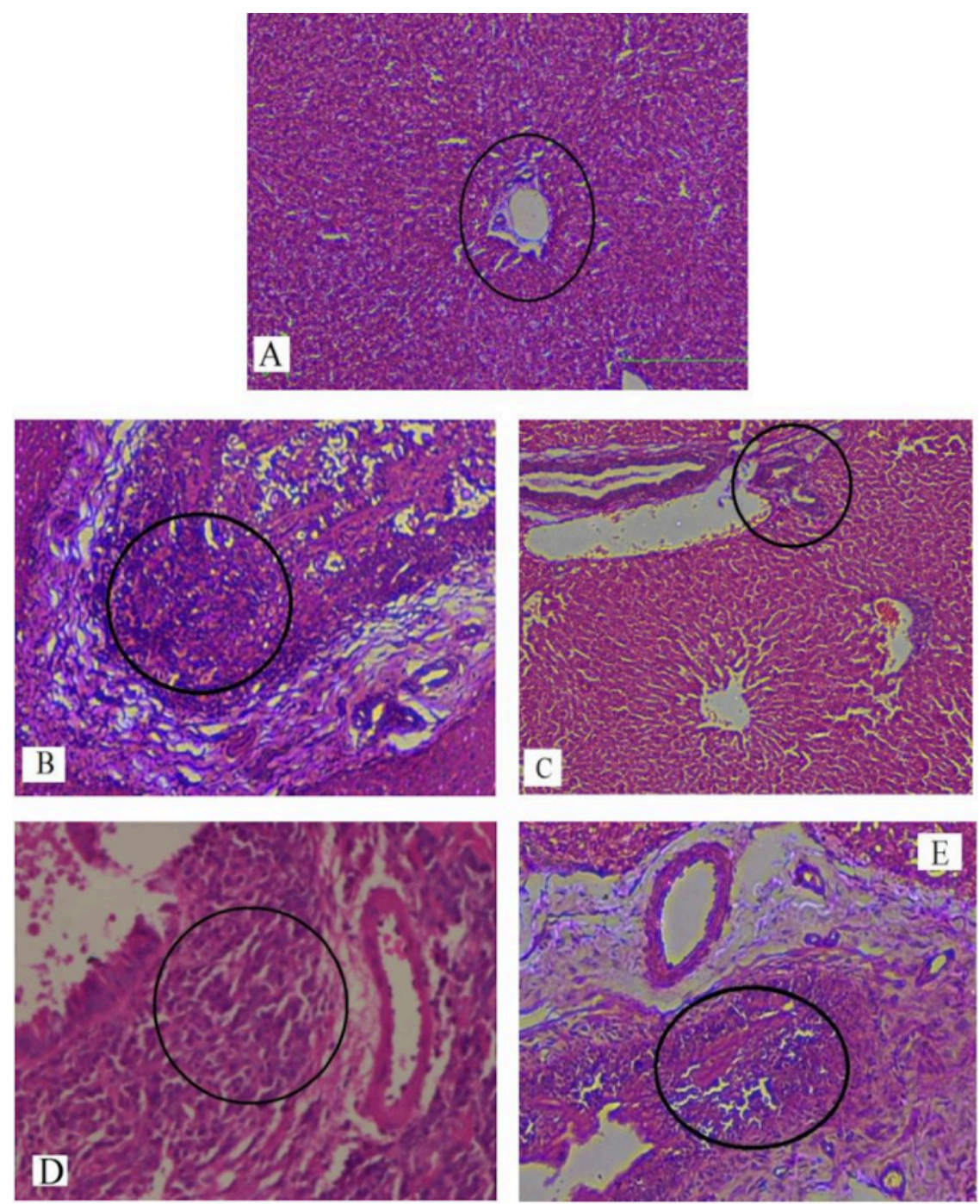

Figure 7. H\&E staining of liver sections. H\&E stained sections of liver tissue shows severe (group B, Par-CM intoxicated), mild (group C, Po-HF \& Par-CM), severe (group D, As-Ac \& Par-CM), moderate (group E, Po-HF + As-AC \& Par$\mathrm{CM}$ ) infiltration of inflammatory cells. Group (A, negative control) shows normal portal area. The rate of inflammatory cells infiltration indicates the intensity of hepatic challenge to a foreign intoxicant.

In our study, elevated levels of all the aforementioned bio-indicators were observed in hepatic damage. The measure of ALT, AST, ALP and total bilirubin in ParCM intoxicated group $B$ was significantly $(P<0.05)$ higher than in the untreated group $A$ (Table 1, Figs. 1-4), demonstrating the damaging effects of ParCM on hepatic cell membranes from enzymes residing within the liver cells and leaking out of cell boundaries in the bloodstream, as reported by Raja et al. (2007) (Raja et al., 2007) and Jain \& Singha (2012) (Jain and Singhai, 2012). Our results show similar liver damaging effects of ParCM, as which are in accordance with studies by Ahmad et al. (2010), Rehman et al. (2013), Naveed and Ibrar 
(2014), Rehman et al. (2015), and Mumtaz et al. (2015) (Ahmad et al., 2010; Mumtaz et al., 2015; Naveed and Ibrar, 2014; Rehman et al., 2015; Rehman et al., 2013). The hepatotoxic effects of ParCM were further verified by histopathological analyses. Our findings revealed a severe congestion of the central vein and sinusoids, infiltration of inflammatory cells, and periportal fibrosis in liver tissues samples of group B (Figs. 5-8). Similar histopathological observations with high dose ParCM have also been reported (Naveed and Ibrar, 2014; Rehman et al., 2015; Rehman et al., 2013).
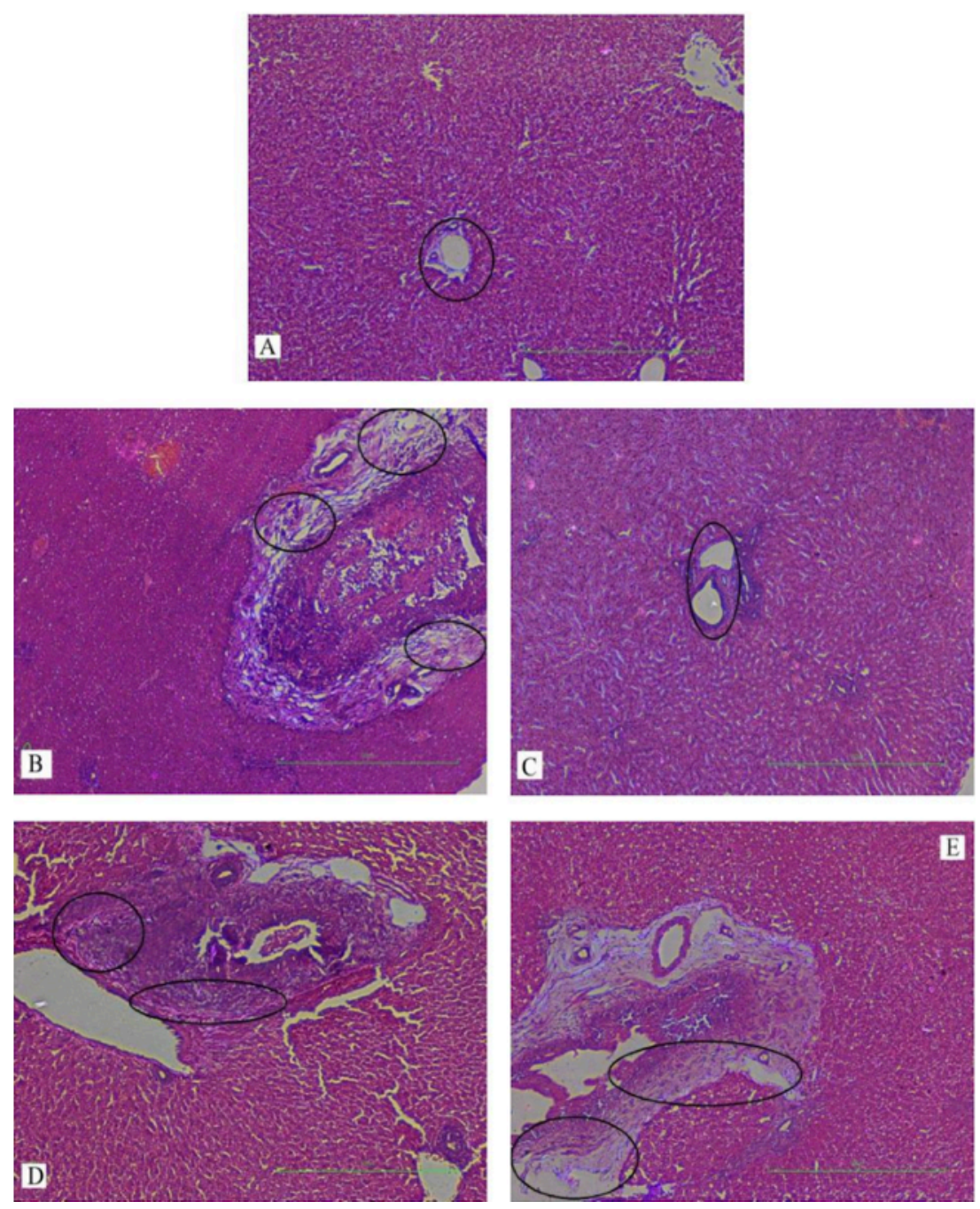

Figure 8. H\&E staining of liver sections. H\&E stained sections of liver tissue shows severe (group B, Par-CM intoxicated), severe (group D, As-Ac \& Par-CM), severe (group E, Po-HF + As-AC \& Par-CM) periportal fibrosis. Groups ( $\mathrm{A}$, negative control) \& (group $\mathrm{C}, \mathrm{Po}-\mathrm{HF} \&$ Par-CM) shows no phenomenon of fibrosis. The volume of connective tissue deposition in periportal area explains the severity of hepatic injury. 
The PoHF used in the study for evaluation of hepatoprotective effects caused a significant decline in serum levels of liver enzymes and total bilirubin, compared to levels for group $B$, indicating that the herbal product positively prevented the deteriorating effects of ParCM on the liver (Table. 1, Figs. 1-4). Histopathological outcomes also reinforced the results of the biochemical analyses; central vein \& sinusoids were mildly congested, infiltration of inflammatory cells was also of mild nature and no phenomenon of fibrosis was observed in group C (Figs. 5-8). The composition of PoHF includes different parts of natural plants and the beneficial effects achieved in our study may be attributed to the various constituents contained in the herbs of herbal formulation.

In group D (AsAc treatment), the level of liver biomarkers was significantly $(P<$ 0.05) greater than that for group $B$ and for group $C$, indicating severe disturbance in the transport mechanisms and permeability of the liver cell membranes (Table 1, Figs. 1-4). The biochemical analysis data was fully supported by the histopathological results, as witnessed by the severe congestion of central vein \& sinusoids, severe infiltration of inflammatory cells and periportal fibrosis in group D (Figs. 5-8). Indeed, Richardson and Ponka (1997) found that in spite of its role as a prototypical antioxidant, AsAc also exhibited pro-oxidant characteristics in different situations (Richardson and Ponka, 1997). Halliwell and Gutteridge (1986) reported that the pro-oxidant action of AsAc depends on the presence of catalytic forms of metals (Halliwell and Gutteridge, 1986). It was explained by Halliwell (1996) (Halliwell, 1996) and Buettner and Jurkiewicz (1996) (Buettner and Jurkiewicz, 1996) that if catalytic metal ions are available in excess as is the case in pathological conditions, ascorbic acid reduces the transition metal ions. This results in excessive production of ferrous ions which by interacting with hydrogen peroxide in Fenton reaction creates more hydroxyl radicals, leading to damaging effects. Bauer et al. (2000) found that in ParCM-poisoned liver, heme oxygenase, an enzyme responsible for breakdown of heme from hemoglobin and ultimately liberation of more free iron, is upregulated (Bauer et al., 2000). In light of this finding, it may be postulated that in our study due to intoxication with high dose of ParCM in group $D$, the activity of heme oxygenase might have accelerated resulting in frequent availability of free transition iron and presence of AsAc, causing reduction of transition metal ions, boost of the Fenton reaction, and detrimental effects on the liver cells. Wang et al. (2007), Park and Lee (2008), Kamel et al. (2010), and Marim et al. (2015) have all reported the damaging effects of AsAc in their studies (Kamel et al., 2010; Marim et al., 2015; Park and Lee, 2008; Wang et al., 2007).

The values of ALT, AST, ALP and total bilirubin in the blood serum of the animals of group $E$ were significantly greater than group C (Table 1, Figs. 1-4). Microscopy of liver sections of group $E$ showed moderate congestion of central vein \& sinusoids, infiltration of inflammatory cells and severe fibrosis (Figs. 5-8), thereby corroborating the results obtained in biochemical analyses of blood serum. Although PoHF alone showed hepatoprotective effects in group $\mathrm{C}$, here 
in group $\mathrm{E}$ (where PoHF \& AsAc were co-administered) liver protection was not achieved. AsAc exhibited liver-damaging effects in group D; similar effects seen in group $\mathrm{E}$ may be ascribed to AsAc in ParCM poisoned animals.

Overall, administration of PoHF alone exhibited a remarkable hepatic cell protection in ParCM- intoxicated animals. Oral administration of AsAc, in a ParCM poisoned rabbit animal model, surprisingly resulted in more malicious effects. Co-administration of PoHF and AsAc (ideally to achieve synergistic effects to counter ParCM intoxication) was not achieved. This may be due to the possibility that AsAc transitions from an antioxidant to pro-oxidant in ParCMintoxicated liver, thereby turning the AsAc from a beneficial to a noxious element.

\section{Conclusion}

PoHF is a successful hepatoprotective regimen in animals, even those administered with high dose ParCM. In our study, AsAc failed to generate hepatic cell protective effects and concomitant administration of PoHF with AsAc showed no benefit for ParCM-poisoned rabbits. Further studies of other hepatotoxicity inducers (besides ParCM) are warranted. Moreover, dose studies of AsAc and ParCM using a larger sample size and in other animal models are warranted.

\section{Abbreviations}

As-Ac: Ascorbic acid; Par-CM: Paracetamol; Po-HF: Polyherbal formulation; AST: Aspartate aminotransferase; ALT: Alanine aminotransferase; ALP: Alkaline phosphatase; CMC: Carboxy Methyl Cellulose; H \& E: Hematoxylin \& Eosin

\section{Author contribution}

Muhammad Fiaz designed the study, collected the literature, executed the experimental work and wrote the manuscript. Waseem Mehmood biochemically analyzed the blood serum. Ghulam Mustafa and Abdul Rauf performed the processing of the liver tissues, examined the slides of liver samples under microscope and concluded the findings of histopathology. Komal Najam reviewed the final manuscript. Naghma Fiaz statistically analyzed the data. Lubna Shakir and Alamgeer supervised the study. 


\section{References}

Achliya, G.S., Wadodkar, S.G., and Dorle, A.K. (2004). Evaluation of hepatoprotective effect of Amalkadi Ghrita against carbon tetrachloride-induced hepatic damage in rats. Journal of Ethnopharmacology 90, 229-232.

Ahmad, M., Bhatti, A.S.A., Maryam, S., Afzal, S., Ahmad, M., and Gillani, A.N. (2010). Hepatoprotective Evaluation of Butea monosperma against Liver damage by Paracetamol in Rabbits. Annals of King Edward Medical University 16.

Akhtar, M.S., Asjad, H.M.M., Bashir, S., Malik, A., Khalid, R., Gulzar, F., and Irshad, N. (2013). Evaluation of antioxidant and hepatoprotective effects of Khamira Gaozaban Ambri Jadwar Ood Saleeb Wala (KGA). Bangladesh Journal of Pharmacology 8, 44-48.

Arsul, V.A., Wagh, S.R., and Mayee, R. (2011). Hepatoprotective activity of livergen, a polyherbal formulation against carbon tetrachloride induced hepatotoxicity in rats. Int $J$ Pharma Pharmac Sci 3, 228-231.

Bauer, I., Vollmar, B., Jaeschke, H., Rensing, H., Kraemer, T., Larsen, R., and Bauer, M. (2000). Transcriptional activation of heme oxygenase-1 and its functional significance in acetaminophen-induced hepatitis and hepatocellular injury in the rat. Journal of hepatology 33, 395-406.

Bonkovsky, H.L., Kane, R.E., Jones, D.P., Galinsky, R.E., and Banner, B. (1994). Acute hepatic and renal toxicity from low doses of acetaminophen in the absence of alcohol abuse or malnutrition: evidence for increased susceptibility to drug toxicity due to cardiopulmonary and renal insufficiency. Hepatology 19, 1141-1148.

Buettner, G.R., and Jurkiewicz, B.A. (1996). Catalytic metals, ascorbate and free radicals: combinations to avoid. Radiation research 145, 532-541.

Carleton, H., Drury, R., and Wallington, E. (1967). Carleton's histological technique. Oxford University Press. New York.

Duarte, T.L., and Lunec, J. (2005). Review: When is an antioxidant not an antioxidant? A review of novel actions and reactions of vitamin C. Free radical research 39, 671-686.

Feroz, Z., and Khan, R.A. (2013). Hepatoprotective effect of herbal drug on CCl 4 induced liver damage. Pakistan journal of pharmaceutical sciences 26.

Ghasemzadeh, A., Jaafar, H.Z., and Rahmat, A. (2010). Antioxidant activities, total phenolics and flavonoids content in two varieties of Malaysia young ginger (Zingiber officinale Roscoe). Molecules 15, 4324-4333.

Girish, C., Koner, B., Jayanthi, S., Rao, K., Rajesh, B., and Pradhan, S. (2009). Hepatoprotective activity of six polyherbal formulations in paracetamol induced liver toxicity in mice.

Halliwell, B. (1996). Commentary: Vitamin C: Antioxidant or Pro-Oxidant In Vivo? Free radical research 25, 439-454.

Halliwell, B., and Gutteridge, J.M. (1986). Oxygen free radicals and iron in relation to biology and medicine: some problems and concepts. Archives of biochemistry and biophysics 246, 501-514.

Ingawale, D.K., Shah, P.V., and Patel, S.S. (2015). Hepatoprotective effect of virgoliv syrup against ccl4 induced hepatic injury in rats. 2015, 6.

Iweala, E.E.J., Obichi, I., and Omotosho, O. (2011). Biochemical and histological responses of hepatotoxic rats fed Musa paradisiaca L. supplemented diet. International Journal of Pharmacology 4, 471-477. 
Jain, N.K., and Singhai, A.K. (2012). Ameliorative effects of Spinacia oleracea L. seeds on carbon tetrachloride (CCl4)-induced hepatotoxicity: in vitro and in vivo studies. Asian Pacific Journal of Tropical Biomedicine 2, S232-S237.

Jollow, D., Mitchell, J., Potter, W., Davis, D., Gillette, J., and Brodie, B. (1973). Acetaminophen-induced hepatic necrosis. II. Role of covalent binding in vivo. Journal of Pharmacology and Experimental Therapeutics 187, 195-202.

Kamel, H., Azza, H., Walaa, A., Ahmed, M., and Mohamed, A. (2010). Protective effect of some antioxidants against $\mathrm{CCl} 4$-induced toxicity in liver cells from BRL3A cell line. J Am Sci 6, 992-1003.

Kang, K. (2002). Mechanism of hepatic ischemia/reperfusion injury and protection against reperfusion injury. Paper presented at: Transplantation Proceedings (Elsevier).

Lee, S.S., Buters, J.T., Pineau, T., Fernandez-Salguero, P., and Gonzalez, F.J. (1996). Role of CYP2E1 in the hepatotoxicity of acetaminophen. Journal of Biological Chemistry 271, 12063-12067.

Makin, A.J., and Williams, R. (1997). Acetaminophen-induced hepatotoxicity: predisposing factors and treatments. Adv Intern Med 42, 453-483.

Marim, R.G., Gusmao, A.S.d., Castanho, R.E.P., Deminice, R., Therezo, A.L.S., Jordao Junior, A.A., Assis, M.R.d., Taipeiro, E.d.F., and Martins, L.P.A. (2015). Effects of vitamin C supplementation on the chronic phase of Chagas disease. Revista do Instituto de Medicina Tropical de São Paulo 57, 245-250.

Mitchell, J.R., Thorgeirsson, S.S., Potter, W.Z., Jollow, D.J., and Keiser, H. (1974). Acetaminophen-induced hepatic injury: Protective role of glutathione in man and rationale for therapy. Clinical Pharmacology \& Therapeutics 16, 676-684.

Monsef, H.R., Ghobadi, A., Iranshahi, M., and Abdollahi, M. (2004). Antinociceptive effects of Peganum harmala L. alkaloid extract on mouse formalin test. J Pharm Pharm Sci 7, 65-69.

Mukherjee, P.K., Wahile, A., Kumar, V., Rai, S., Mukherjee, K., and Saha, B. (2006). Marker profiling of botanicals used for hepatoprotection in Indian system of medicine. Drug information journal 40, 131-139.

Mumtaz, A., Ch, M.Z., and Shah, S.N.H. (2015). Screening of hepatoprotective effect of methanolic extract of Solanum nigrum against paracetamol. Pakistan Journal of Pharmaceutical Research 1, 70-77.

Nadeem, M., Dandiya, P., Pasha, K., Imran, M., Balani, D., and Vohora, S. (1997). Hepatoprotective activity of Solanum nigrum fruits. Fitoterapia 68, 245-251.

Naveed, S., and Ibrar, M. (2014). Hepatoprotective activity of iphiona grantioides and pluchea arguta for acetaminophen induced toxicity in rabbits. Pakistan Journal of Weed Science Research 20.

Neraliya, S., and Gaur, R. (2004). Juvenoid activity in plant extracts against filarial mosquito Culex quinquefasciatus. Journal of Medicinal and Aromatic Plant Sciences 26, 34-38.

Park, S.-W., and Lee, S.-M. (2008). Antioxidant and prooxidant properties of ascorbic acid on hepatic dysfunction induced by cold ischemia/reperfusion. European journal of pharmacology 580, 401-406.

Parmar, S.R., Vashrambhai, P.H., and Kalia, K. (2010). Hepatoprotective activity of some plants extract against paracetamol induced hepatotoxicity in rats. J Herbal Med Toxicol 4, 101-106. 
Raja, S., Ahamed, K.N., Kumar, V., Mukherjee, K., Bandyopadhyay, A., and Mukherjee, P.K. (2007). Antioxidant effect of Cytisus scoparius against carbon tetrachloride treated liver injury in rats. Journal of ethnopharmacology 109, 41-47.

Rehman, J.U., Aktar, N., Khan, M.Y., Ahmad, K., Ahmad, M., Sultana, S., and Asif, H.M. (2015). Phytochemical screening and hepatoprotective effect of Alhagi maurorum boiss (Leguminosae) against paracetamol-induced hepatotoxicity in rabbits. Tropical Journal of Pharmaceutical Research 14, 1029-1034.

Rehman, J.U., Saqib, N.U., Akhtar, N., Jamshaid, M., Asif, H.M., Sultana, S., and Rehman, R.U. (2013). Hepatoprotective activity of aqueous-methanolic extract of Suaeda fruticosa in paracetamol-induced hepatotoxicity in rabbits. Bangladesh Journal of Pharmacology 8, 378-381.

Richardson, D.R., and Ponka, P. (1997). The molecular mechanisms of the metabolism and transport of iron in normal and neoplastic cells. Biochimica Et Biophysica Acta (BBA)-Reviews on Biomembranes 1331, 1-40.

Sabina, E., Samuel, J., RajappaRamya, S., Patel, S., Mandal, N., Pranatharthiiharan, P., Mishra, P.P., and Rasool, M. (2009). Hepatoprotective and antioxidant potential of Spirulina fusiformis on acetaminophen-induced hepatotoxicity in mice. IJIB 6, 1-5.

Sapakal, V., Ghadge, R., Adnaik, R., Naikwade, N., and Magdum, C. (2008). Comparative hepatoprotective activity of Liv-52 and livomyn against carbon tetrachloride-induced hepatic injury in rats. International Journal of Green Pharmacy 2, 79.

Sharma, A., Chakraborti, K., and Handa, S. (1991). Antihepatotoxic activity of some Indian herbal formulations as compared to silymarin. Fitoterapia 62, 229-235.

Sivakumar, V., Rajan, M.D., Sadiq, A.M., and Rajeshkumar, S. (2014). Hepatoprotective Effect of Polyherbal Formulations in Paracetamol Induced Hepatic Damaged Experimental Rats.

Vermeulen, N., Bessems, J., and Van de Straat, R. (1992). Molecular aspects of paracetamol-induced hepatotoxicity and its mechanism-based prevention. Drug metabolism reviews 24, 367-407.

Wang, C., Liang, J., Zhang, C., Bi, Y., Shi, X., and Shi, Q. (2007). Effect of ascorbic acid and thiamine supplementation at different concentrations on lead toxicity in liver. Annals of Occupational Hygiene 51, 563-569.

Yang, J., Li, Y., Wang, F., and Wu, C. (2010). Hepatoprotective effects of apple polyphenols on CCl4-induced acute liver damage in mice. Journal of agricultural and food chemistry 58, 6525-6531. 